\title{
ON SOME CHARACTERISTIC PROPERTIES OF QUASI-FROBENIUS AND REGULAR RINGS
}

\author{
MASATOSI IKEDA AND TADASI NAKAYAMA
}

Recently the first writer [1] gave a characterization of quasiFrobenius rings, introduced formerly by the second writer [3], in terms of a condition proposed by $\mathrm{K}$. Shoda, which reads: A ring $A$ satisfying minimum condition and possessing a unit element is a quasi-Frobenius ring if and only if $A$ satisfies the following condition: ${ }^{1}$

(a) every ( $A$-left-) homomorphism of a left-ideal of $A$ into $A$ may be given by the right multiplication of an element of $A$.

In the present note we shall offer a simpler ${ }^{2}$ proof of this, making use of the second writer's former characterization of quasi-Frobenius rings and a theorem in a previous joint note of the writers. The present approach starts, contrary to the one in $[1],{ }^{3}$ with a theorem (Theorem 1) which is independent of any chain condition (or is concerned with maximum condition at most (corollary to Theorem 1) and which is perhaps of interest by itself. We shall also show that a remark at the end of [1], concerning semisimple rings with minimum condition, may be freed from chain condition, to yield a certain characterization of von Neumann's regular rings (Theorem 3).

1. Conditions (a), $\left(\mathrm{a}^{*}\right)$. Let $A$ be an (associative) ring. On weakening (a), we consider the condition:

$\left(\mathrm{a}^{*}\right)$ every homomorphism of a finitely generated left-ideal of $A$ into $A$ may be given by the right multiplication of an element of $A$.

On restricting ourselves further to principal left-ideals, we obtain a (further weaker) condition, which we denote by $\left(\mathrm{a}^{* *}\right)$.

On the other hand, with a subset $X$ in $A$, we denote, as in our former papers, the set of left (right) annihilators of $X$ in $A$ by $l(X)$ $(r(X))$. We consider the conditions ${ }^{4}$

Received by the editors June 1, 1953.

1 Under the assumption of the existence of unit element, this is equivalent to Shoda's condition that every homomorphism between two left-ideals should be extended to an endomorphism of $A$ (considered as $A$-left-module).

2 Simpler, provided we used the results in our former works.

${ }^{3}$ On the other hand, the proof in [1] gives some further details for the case with minimum condition.

1 Observe that the dual relations $r\left(\mathfrak{l}_{1}+\mathfrak{l}_{2}\right)=r\left(\mathfrak{l}_{1}\right) \bigcap r\left(\mathfrak{l}_{2}\right)$ is trivial (and is always true). Contrary to our former papers, we use " + " for a mere module sum which is not necessarily direct. 


$$
\begin{aligned}
r\left(\mathfrak{l}_{1} \cap \mathfrak{l}_{2}\right) & =r\left(\mathfrak{l}_{1}\right)+r\left(\mathfrak{l}_{2}\right), \\
r(l(\mathfrak{r})) & =\mathfrak{r},
\end{aligned}
$$

concerning, respectively, pairs of left-ideals $\mathfrak{l}_{1}, \mathfrak{l}_{2}$ of $A$ and right-ideals $\mathfrak{r}$ of $A$. We denote by $\left(\mathrm{b}^{*}\right),\left(\mathrm{c}^{*}\right)$ the conditions which demand the validity of (b), (c), respectively, for finitely generated left- or rightideals. On restricting (c) further to principal right-ideals we introduce the condition $\left(\mathrm{c}^{* *}\right)$.

TheOREM 1. Let $A$ be a ring with unit element. Then the following implications hold in $A$ :

(i) $\left(\mathrm{a}^{* *}\right) \leftrightarrow\left(\mathrm{c}^{* *}\right)$;

(ii) $\left(\mathrm{a}^{*}\right) \leftrightarrow\left(\mathrm{b}^{*}\right),\left(\mathrm{c}^{* *}\right)$;

(iii) (a) $\rightarrow\left(\right.$ b),$\left(c^{*}\right)$.

Proof. ${ }^{5}$ (i) Assume $\left(\mathrm{a}^{* *}\right)$. We consider a principal left-ideal $A a$. It is ( $A$-left-)isomorphic to the residue-module $A / l(a)$ by the correspondence $x(\bmod l(a)) \leftrightarrow x a$. Let $b$ be an arbitrary element in $r(l(a))$. As $l(b) \supseteq l(a)$, the mapping $x a \rightarrow x b$ gives a homomorphism of $A a$ onto $A b$. According to $\left(\mathrm{a}^{* *}\right)$ there must exist an element $c$ in $A$ satisfying $a c=b$, and hence $b \in a A$. This shows $r(l(a A))(=r(l(a)))$ ( $\subseteq$ whence) $=a A$.

Assume conversely (c**). Let $A a$ be a principal left-ideal in $A$ and let $\theta$ be a homomorphism of $A a$ into $A$. Clearly $l(a) \subseteq l\left(a^{\theta}\right)$. Hence, by $\left(\mathrm{c}^{* *}\right), a^{\theta} A=r\left(l\left(a^{\theta} A\right)\right) \subseteq r(l(a A))=a A$, and there exists an element $c$ with $a^{\theta}=a c$. The right-multiplication of $c$ onto $A a$ gives the homomorphism $\theta$.

(ii) Assume $\left(a^{*}\right)$. Let $\mathfrak{l}_{1}, \mathfrak{l}_{2}$ be two finitely generated left-ideals and let $b$ be an arbitrary element in $r\left(\mathfrak{l}_{1} \cap \mathfrak{l}_{2}\right)$. Taking an element $c$ of $A$ arbitrarily, ${ }^{6}$ we consider the homomorphic mappings

$$
\begin{array}{lll}
\theta_{1}: & x_{1} \rightarrow x_{1} c & \left(x_{1} \in \mathfrak{l}_{1}\right), \\
\theta_{2}: & x_{2} \rightarrow x_{2}(c+b) & \left(x_{2} \in \mathfrak{l}_{2}\right)
\end{array}
$$

of $\mathfrak{l}_{1}$ and $\mathfrak{l}_{2}$ onto $\mathfrak{l}_{1} c$ and $\mathfrak{l}_{2}(c+b)$, respectively. As $b \in r\left(\mathfrak{l}_{1} \cap \mathfrak{l}_{2}\right)$, these mappings $\theta_{1}, \theta_{2}$ coincide on $\mathfrak{l}_{1} \cap \mathfrak{l}_{2}$ and together generate a homomorphic mapping $\theta$ of $\mathfrak{l}_{1}+\mathfrak{l}_{2}$ onto $\mathfrak{l}_{1} c+\mathfrak{l}_{2}(c+b)$ :

$$
x_{1}+x_{2} \rightarrow x_{1} c+x_{2}(c+b) \text {. }
$$

By $\left(\mathrm{a}^{*}\right)$ this homomorphism $\theta$ must be given by the right-multiplication of an element, say $a$, and we must have thus

5 A half of our proof of (i), (ii) is a mere reproduction of the arguments in [1]. The other half is to follow them in the reverse order.

- We may take for instance the unit element for $c$. 


$$
c-a \in r\left(\mathfrak{l}_{1}\right), \quad(c+b)-a \in r\left(\mathfrak{l}_{2}\right) .
$$

Here $b=(c+b-a)-(c-a) \in r\left(\mathfrak{l}_{1}\right)+r\left(\mathfrak{l}_{2}\right)$. This shows that $r\left(\mathfrak{l}_{1} \cap \mathfrak{l}_{2}\right)$ $(\subseteq$ whence $)=r\left(\mathfrak{l}_{1}\right)+r\left(\mathfrak{l}_{2}\right)$, proving $\left(\mathrm{b}^{*}\right)$. That $\left(\mathrm{c}^{* *}\right)$ holds has been secured in (i).

Assume conversely $\left(\mathrm{b}^{*}\right)$ and $\left(\mathrm{c}^{* *}\right)$. Then $\left(\mathrm{a}^{* *}\right)$ holds as has been seen in (i). Let $\mathfrak{l}=A a_{1}+A a_{2}+\cdots+A a_{n}$ be any finitely generated left-ideal in $A$, and suppose that $\left(\mathrm{a}^{*}\right)$ is the case for its left-subideal $\mathfrak{l}_{1}=A a_{1}+A a_{2}+\cdots+A a_{n-1}$. Let $\theta$ be a homomorphism of $\mathfrak{l}$ into $A$, and let $\theta_{1}, \theta_{2}$ be the restrictions of $\theta$ to $\mathfrak{l}_{1}, A a_{n}$ respectively. Each of $\theta_{1}, \theta_{2}$ is given by the right-multiplication of an element of $A$, say $c_{1}$ or $c_{2}$ respectively. As $\theta_{1}$ and $\theta_{2}$ coincide on $\mathfrak{l}_{1} \cap A a_{n}$, we have $c_{1}-c_{2}$ $\in r\left(\mathfrak{l}_{1} \cap A a_{n}\right)$. The last right-ideal is, by $\left(\mathrm{b}^{*}\right)$, equal to $r\left(\mathfrak{l}_{1}\right)+r\left(A a_{n}\right)$. Thus $c_{1}-c_{2}=b_{1}-b_{2}$ with $b_{1} \in r\left(\mathfrak{l}_{1}\right), b_{2} \in r\left(A a_{n}\right)$. Denote $c_{1}+b_{1}\left(=c_{2}+b_{2}\right)$ by $c$. Then the right-multiplication of $c$ onto $\mathfrak{l}\left(=\mathfrak{l}_{1}+A a\right)$ gives our homomorphism $\theta$. Now $\left(\mathrm{a}^{*}\right)$ can generally be established by induction.

(iii) Assume (a). On repeating our consideration in the first half of (ii), this time with arbitrary left-ideals $\mathfrak{l}_{1}, \mathfrak{l}_{2}$, we see the validity of (b). Combining that with $\left(\mathrm{c}^{* *}\right)$ (already established in (i)) we have

$$
\begin{aligned}
r\left(l\left(a_{1} A+a_{2} A+\cdots+a_{n} A\right)\right) & \\
& =r\left(l\left(a_{1} A\right) \cap l\left(a_{2} A\right) \cap \cdots \cap l\left(a_{n} A\right)\right) \\
& =r\left(l\left(a_{1} A\right)\right)+r\left(l\left(a_{2} A\right)\right)+\cdots+r\left(l\left(a_{n} A\right)\right) \\
& =a_{1} A+a_{2} A+\cdots+a_{n} A
\end{aligned}
$$

for any finitely generated right-ideal $\mathfrak{r}=a_{1} A+a_{2} A+\cdots+a_{n} A$, proving $\left(c^{*}\right)$.

Corollary. Let $A$ be a ring with unit element and satisfying maximum condition. Then $A$ satisfies (a) (equivalently $\left(\mathrm{a}^{*}\right)$ ) if and only if the correspondence $\mathfrak{l} \rightarrow r(\mathfrak{l}) \quad(\mathfrak{l}:$ left-ideal of $A$ ) gives a (lattice-theoretical) dual-homomorphism of the left-ideal lattice of $A$ onto the right-ideal lattice of $A$.

Proof. In our $A$ the conditions (a), (a*) are equivalent and (b), $\left(b^{*}\right)$ are equivalent. (ii) and (iii) of Theorem 1 together give our corollary.

2. Quasi-Frobenius rings. Let now $A$ be a ring with unit element satisfying minimum condition (whence maximum condition too). Our Theorem 1 (or its corollary), combined with Theorem 6 of [3], shows that $A$ satisfies (a) if it is quasi-Frobeniusean. To prove the converse, assume that $A$ satisfies (a). Then, by Theorem 1 (or its corol- 
lary), we have (c) in $A$. Hence, according to Theorem 1 of $[2]^{7}$; there exists for any maximal left-ideal $\mathfrak{l}_{1}$ in $A$ a minimal left-ideal $\mathfrak{l}_{2}$ in $A$ with $A / \mathfrak{l}_{1} \cong \mathfrak{l}_{2}$. Let now $\mathfrak{l}$ be an arbitrary left-ideal in $A$. We want to show that $\mathfrak{l}=l(r(\mathfrak{l}))$. To do so, assume the contrary (i.e. $\mathfrak{l} \subset l(r(\mathfrak{l}))$ ) and let $\mathfrak{I}_{0}$ be a left-subideal of $l(r(\mathfrak{l}))$ in which $\mathfrak{l}$ is maximal. There exists (a maximal left-ideal $\mathfrak{l}_{1}$ of $A$ such that $\mathfrak{l}_{0} / \mathfrak{l} \cong A / \mathfrak{l}_{1}$, whence there exists) a minimal left-ideal $\mathfrak{l}_{3}$ of $A$ isomorphic to $\mathfrak{I}_{0} / \mathfrak{l}$. Thus we have a homomorphism of $\mathfrak{l}_{0}$ onto $\mathfrak{l}_{3}$, mapping $\mathfrak{l}$ onto 0 , which must be, by (a), given by the right-multiplication of an element, say $c$. We have $\mathfrak{l} c=0$, i.e. $c \in r(\mathfrak{l})$, whence $\mathfrak{l}_{0} \subseteq l(r(\mathfrak{l})) \subseteq l(c), \mathfrak{l}_{0} c=0$, contrary to $\mathfrak{l}_{0} c=\mathfrak{l}_{3}$. Thus necessarily $\mathfrak{l}=l(r(\mathfrak{l}))$ for any left-ideal $l$ in A. This and (c) show, again by Theorem 6 of [3], that $A$ is quasi-Frobeniusean. Thus we have

TheOREM 2. $A$ ring $A$ with unit element and satisfying minimum condition is quasi-Frobeniusean if and only if it satisfies (a).

\section{Regular rings.}

Theorem 3. A ring $A$ with unit element is regular if and only if it satisfies the following condition:

$\left(\mathrm{a}_{0}^{*}\right)$ every ( $A$-left-) homomorphism of a principal (or finitely generated) left-ideal $\mathfrak{l}$ of $A$ into (or onto) a residue-module $A / \mathfrak{l}^{\prime}$, of $A$ modulo a left-ideal $\mathfrak{l}^{\prime}$, may be obtained by the right-multiplication of an element, say $c$, of $A: x \rightarrow x c\left(\bmod \mathfrak{l}^{\prime}\right)(x \in \mathfrak{l})$.

Proof. Suppose first that $\left(\mathrm{a}_{0}^{*}\right)$ is the case. With an arbitrary element $a$ in $A$, set $\mathfrak{l}^{\prime}=l(a)$. The isomorphism $y a \rightarrow y\left(\bmod \mathfrak{l}^{\prime}\right)(y \in A)$ of $A a$ onto $A / \mathfrak{l}^{\prime}$ must be obtained by the right-multiplication of an element, say $c$, of $A$. Thus $y \equiv y a c \bmod \mathfrak{l}^{\prime}$, whence $y a=y a c a$, for any element $y$ in $A$. So $a=a c a$, and $A$ is a regular ring.

Assume conversely that $A$ is a regular ring. (Any finitely generated right-ideal is principal and) any principal right-ideal $a A$ is generated by an idempotent element, say $e$. Let $e=a b$. We have $y \equiv y e=y a b$ $\bmod l(a)(=l(a A)=l(e))$ for any $y \in A$. If we have a homomorphism of $A a$ into a residue-module $A / \mathfrak{l}^{\prime}$ and if the image of $a$ is $a_{1}\left(\bmod \mathfrak{l}^{\prime}\right)$, then we ought to have $l(a) a_{1} \subseteq \mathfrak{l}^{\prime}$ and $(y-y a b) a_{1} \subseteq \mathfrak{l}^{\prime}$, i.e., $y a_{1}$ $\equiv y a b a_{1} \bmod \mathfrak{I}^{\prime}$ for any $y \in A$. Thus our homomorphism of $A a$ into $A / \mathfrak{l}^{\prime}$ is given by $y a \rightarrow y a c(y \in A)$ with $c=b a_{1}$, which proves $\left(\mathrm{a}_{0}^{*}\right)$.

\section{REFERENCES}

1. M. Ikeda, A characterization of quasi-Frobenius rings, Osaka Math. J. vol. 4 (1952) pp. 203-210.

${ }^{7}$ To use this theorem is more or less equivalent to using Lemma 2 in [1]. 
2. M. Ikeda and T. Nakayama, Supplementary remarks on Frobeniusean algebras, II, Osaka Math. J. vol. 2 (1950) pp. 7-12.

3. T. Nakayama, On Frobeniusean algebras, II, Ann. of Math. vol. 42 (1941) pp. $1-21$.

OSAKa UNIVERSITY AND

NAGOYA UNIVERSITY

\section{THE NUMBER OF SUBGROUPS OF GIVEN INDEX IN NONDENUMERABLE ABELIAN GROUPS}

\section{W. R. SCOTT}

Let $G$ be an Abelian group of order $A>\boldsymbol{\aleph}_{0}$. It has been shown [4, Theorem 9] that there exist $2^{A}$ subgroups of $G$ of order $A$, and that the intersection of all such subgroups is 0 . In this paper, this result is improved to the following: If $\boldsymbol{\aleph}_{0} \leqq B \leqq A$ and $A>\boldsymbol{\aleph}_{0}$, then an Abelian group of order $A$ has $2^{A}$ subgroups of index $B$, and the intersection of all such subgroups is 0 . In addition, it is shown that there is a set of $2^{A}$ subgroups $H_{\alpha}$ of index $B$ such that $G / H_{\alpha} \cong G / H_{\alpha^{\prime}}$ for all $\alpha, \alpha^{\prime}$.

Baer [1, p. 124] showed that if $G$ is an Abelian p-group which is the direct sum of $A$ cyclic groups of bounded order, then $G$ has $2^{A}$ subgroups of index $p$ (here $A$ may equal $\aleph_{0}$ ). The proof in the present paper is accomplished by extending Baer's result in an obvious manner to a wider class of $p$-groups, and then reducing all other cases to this one.

We shall use + and $\sum$ to denote direct sums, and $o(S)$ to denote the number of elements in $S$.

Lemma. Let $H \neq 0$ be an Abelian group, and let $G=\sum H_{\alpha}, \alpha \in S$, $H_{\alpha} \cong H$ for all $\alpha \in S, o(S)=A \geqq \boldsymbol{N}_{0}$. Then there are at least $2^{A}$ subgroups $K_{\beta}$ of $G$ such that $G / K_{\beta} \cong H$.

Proof. (This proof is the same as Baer's, and is included only for the sake of completeness.) Identify $H_{\alpha}$ with $H$. Let $\epsilon_{\alpha}$ be 0 or 1 for each $\alpha, \alpha \in S$. Let $K$ be the set of elements of $G$ such that $h_{\alpha_{0}}=\sum \epsilon_{\alpha} h_{\alpha}$, $\alpha \neq \alpha_{0}$. Then it is easy to verify that $K$ is a subgroup of $G$ and $G=H_{\alpha_{0}}$ $+K$. If $\epsilon_{\alpha}=0$, then $H_{\alpha} \subset K$, but if $\epsilon_{\alpha}=1$, then $H_{\alpha} \cap K=0$. Thus all of the $K^{\prime}$ 's are distinct, and the lemma is proved.

Presented to the Society, September 3, 1953; received by the editors March 2, 1953. 